\title{
Patent Foramen Ovale as a Risk Factor for Cryptogenic Brain Abscess: Case Report and Review of the Literature
}

\author{
Yohsuke Horiuchi, Yuji Kato, Tomohisa Dembo, Hidetaka Takeda, \\ Takuya Fukuoka and Norio Tanahashi
}

\begin{abstract}
We encountered a patient with brain abscess presumably caused by dental infection. The patient displayed patent foramen ovale (PFO) and a giant Eustachian valve, through which spontaneous right-to-left shunt was revealed by transesophageal echocardiography. Reviewing the literature, we find additional cases where brain abscess originated from an increased amount of flora commonly found in the oral cavity that bypassed the pulmonary vascular bed and the lymphatic system through PFO. Additionally, a Eustachian valve should be considered an adjunctive risk factor for initiating a spontaneous right-to-left shunt and predisposing cryptogenic brain abscess in patients with PFO.
\end{abstract}

Key words: brain abscess, patent foramen ovale, Eustachian valve, transesophageal echocardiography

(Intern Med 51: 1111-1114, 2012)

(DOI: 10.2169/internalmedicine.51.7133)

\section{Introduction}

Brain abscess is a relatively uncommon but potentially devastating disease. Rapid identification of the infectious source and knowledge of the spreading mechanism are essential for the initiation of an effective treatment and they influence the patients' clinical outcome (1). Brain abscesses frequently arise from a contiguous source of infection, the hematogenous spread from a distant focal infection, or cranial trauma. However, in $20-30 \%$ of cases, the primary infectious source remains unknown (2). In some of these patients a patent foramen ovale (PFO) or a pulmonary arteriovenous fistula have been found $(3,4)$, possibly allowing bacteria to bypass the pulmonary circulation through a shunt. Some patients with a PFO also have a residual Eustachian valve (EV), an embryological remnant of the inferior vena cava valve that prenatally directs oxygenated blood from the inferior vena cava across PFO into systemic circulation. Even when the EV is prominent, it has generally been considered a benign finding in the absence of associated cardiac anomalies. Recently, it has been reported that EVs may predispose patients with PFO to right-to-left shunt and that this may increase the risk of paradoxical embo- lism (5-7). Here we describe a patient with a PFO whose giant EV caused a spontaneous right-to-left shunt and subsequent brain abscess; we also review the clinical features of brain abscess associated with PFO.

\section{Case Report}

A 55-year old woman was referred to our institute with new onset of aphasia and right hemiparesis. The patient had untreated diabetes and had complained of headache and fever for 1 week prior to admission. Her physical examination was remarkable for poor dental hygiene with numerous dental caries but no evidence of dental abscess. Computed tomographic (CT) scans revealed a hypodense lesion in the left frontal lobe, and magnetic resonance (MR) imaging demonstrated a ring enhancing lesion in the left frontal lobe with edema and mass effect (Fig. 1). A blood culture failed to detect bacteremia; however, the patient had received a systemic intravenous administration of antibiotic at another hospital before she was transferred to our institute. Surgical drainage effectively reduced the mass lesion, and study of the aspirated pus revealed normal flora commonly found in the oral cavity such as Prevotella intermedia, Bacteroides fragilis, and Peptostreptcoccus micros. No further abscesses 


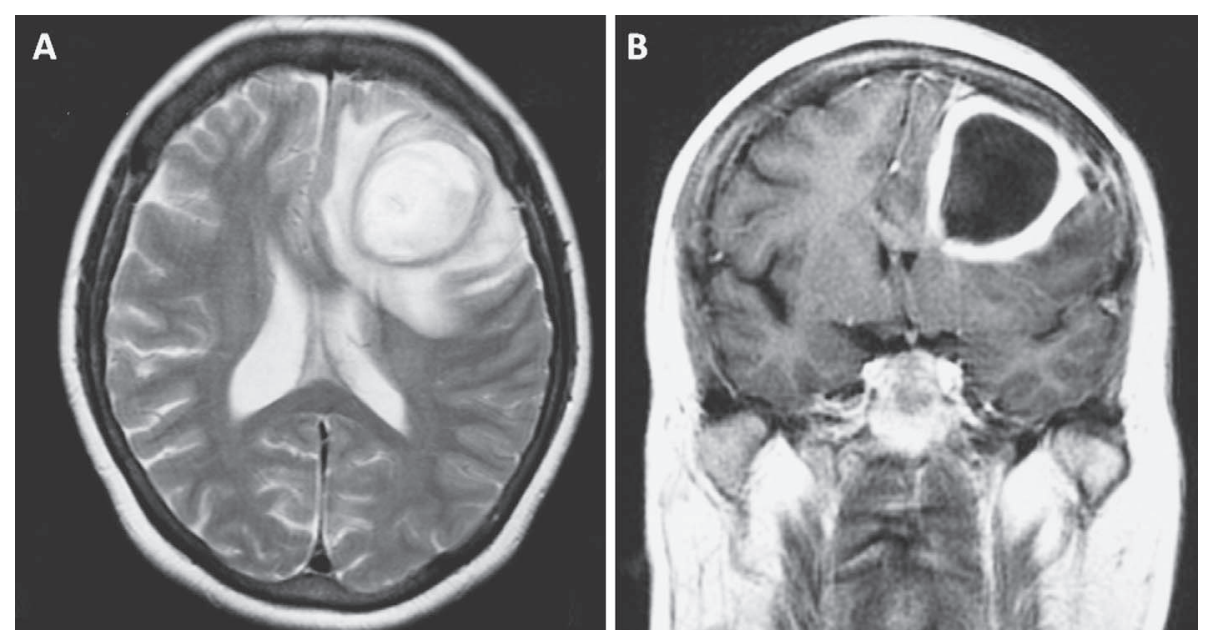

Figure 1. A: T2-weighted axial MR image showing a heterogeneous mass in the left frontal lobe, compressing the left lateral ventricle. B: T1-weighted coronal MR image after gadolinium administration, showing a left frontal hypointense mass lesion with ring enhancement.
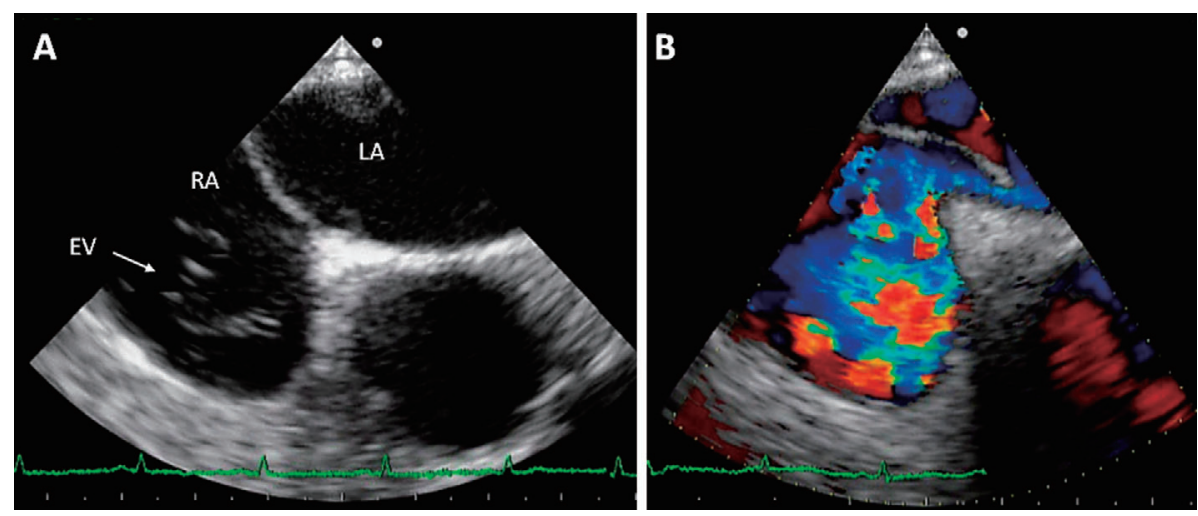

Figure 2. (A) TEE demonstrating PFO and a giant Eustachian valve (EV) as a snake-like mobile structure with a diameter of $>\mathbf{3 0} \mathrm{mm}$. (B) A large right-to-left shunt identified by color Doppler analysis without Valsalva maneuver. RA: right atrium, LA: left atrium

were found on CT scans of the neck, chest, abdomen and pelvis. Notably, a CT scan of the chest did not reveal any pulmonary arteriovenous fistula. Further laboratory and ultrasound diagnostics excluded potential risk factors such as immune deficiency, endocarditis, cyanotic heart disease, and pulmonary hypertension. However, transesophageal echocardiography (TEE) detected a giant persisting EV and PFO, through which a spontaneous right-to-left cardiac shunt was found without Valsalva maneuver (Fig. 2). Subsequent contrast transcranial Doppler detected frequent high-intensity transient signals on the left MCA without Valsalva maneuver. A prominent persisting EV may predispose towards brain abscess formation by providing a passage for infectious emboli from the venous to the arterial circulation through a right-to-left shunt via the PFO. Thus, the brain abscess may have been caused by the hematogeneous spread from a dental infection.

Antibiotic therapy with meropenem was administrated intravenously to the patient for 4 weeks after stereotactic aspiration of the abscess. The patient received warfarin to prevent ischemic stroke but refused percutaneous PFO closure to prevent recurrence of the abscess. She fully recovered and is being followed as an outpatient.

\section{Review of the literature}

There are 12 cases in the literature which have described an association of PFO and brain abscess in adults (Table 1) (4, 8-15). Most of the patients were middle-aged with no significant medical history. Only one patient had a prominent septic source (9). In these cases, most pathogenic bacteria were normally found in the human oral cavity and pharynx. Symptoms ranged from none to focal cranial nerve deficit to lethargy. For all but the lone autopsy case (14), TEE was the diagnostic test of choice and revealed earlyappearing air bubbles in the left atrium, indicative of PFO. In most cases, TEE showed no additional findings, except for single cases of coexisting atrial vegetations (13), atrial septal aneurysm (15) and EV. In this set of cases, most authors hypothesize that the right-to-left shunt through the PFO was the primary contributory factor to brain abscess by permitting infected material to bypass the lungs and enter the systemic circulation $(4,9-12,14,15)$, in contrast to sim- 
Intern Med 51: 1111-1114, 2012 DOI: 10.2169/internalmedicine.51.7133

Table 1. The Literature Which Have Described an Association of PFO and Brain Abscess in Adults

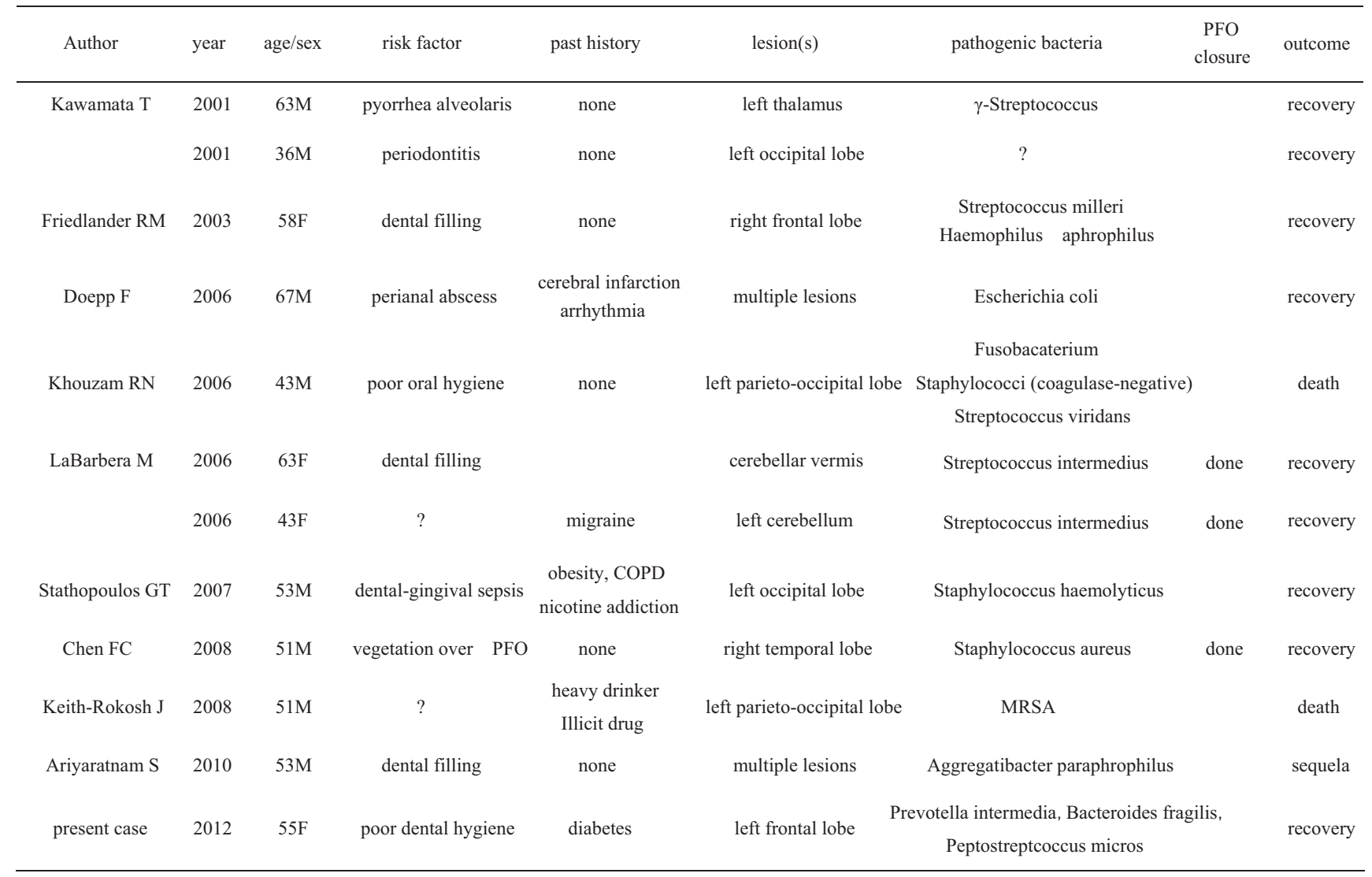

ple seeding of the brain via transit of the infecting bacteria through the valveless emissary veins $(4,9,10)$. This is consistent with our findings in the current case.

\section{Discussion}

Poor dental hygiene has been previously identified as a risk factor for brain abscess. Given that a transient bacteremia may occur in healthy persons from flora normally found in the oral cavity due to simple hygienic procedures such as tooth brushing (16), it is reasonable to believe the bacterial load could be significantly greater in those persons with poor dental hygiene. Normally, these potentially pathogenic organisms are contained within the venous circulation, and would be unable to survive the oxygen-rich environment of the pulmonary vascular bed and the actions of the alveolar macrophages (17). Although some bacteria might escape this first line of defense, they would be eliminated by the lymphatic system. However, a right-to-left shunt would bypass these defenses and allow these organisms to enter the arterial circulation and thus reach the brain. On the basis of the present case and the accompanying literature review, we believe that brain abscess formation in these cases depends on the infectious dose of oral flora and the magnitude of the right-to-left shunt.

We also suggest that the presence of an EV may be a contributing factor in developing a brain abscess from poor dental hygiene. This embryological remnant of the inferior vena cava (IVC) valve normally regresses postnatally (18), and persistence of EV in adults is reported to cause a significant right-to-left shunt in the presence of interatrial communication by altering the blood flow pattern (19). Because blood volume may play a determining role in the development of the arterial septum in the embryologic period (20), we also speculated that a persistent EV may favor PFO by directing blood flow to the area of the fossa ovalis. A persisting EV is a frequent finding in patients with $\operatorname{PFO}(5,6)$. As EV has an average length of $3.6 \mathrm{~mm}$ with a range of $1.5-23 \mathrm{~mm}$ (18), the EV of the current case with a diameter of $>30 \mathrm{~mm}$, is considered giant. Any condition that increases right atrial pressure relative to left can induce paradoxical flow and may result in an embolic event. For example, this can occur, during coughing, sneezing, or straining during a bowel movement. The present case describes a prominent $\mathrm{EV}$, which initiated a spontaneous right-to-left shunt via PFO in the absence of Valsalva maneuver or pulmonary hypertension.

In conclusion, we present a case report and supporting review of the literature wherein a brain abscess originated from an increased amount of oral cavity flora that apparently bypassed the pulmonary vascular bed and the lymphatic system through a PFO. From our findings, we believe EV should be considered an adjunctive risk factor for not only paradoxical embolism but also for cryptogenic brain abscess in patients with PFO. Furthermore, EV may persist more commonly than estimated. We recommend that all pa- 
tients with a brain abscess caused by flora commonly found in the oral cavity should be screened for a PFO and accompanying EV. Conversely, any patient in whom a PFO is identified should be advised to maintain excellent oral hygiene to reduce the risk of brain abscess formation. If a brain abscess associated with a PFO is identified, these patients should undergo surgical evacuation of the abscess in conjunction with prolonged antibiotic therapy until there is no radiographic evidence of a brain abscess and serial blood cultures are negative. Definitive treatment would require closure of the PFO and resection of the EV if necessary, because anticoagulation alone would not prevent a right-to-left shunt of septic emboli.

The authors state that they have no Conflict of Interest (COI).

\section{Acknowledgement}

We are grateful to Drs. Ririko Takeda and Hiroki Kurita of the Department of Cerebrovascular surgery at our center for neurosurgical treatment.

\section{References}

1. Keet PC. Cranial intradural abscess management of 641 patients during the 35 years from 1952 to 1986 . Br J Neurosurg 4: 273278, 1990.

2. Mathisen GE, Johnson JP. Brain abscess. Clin Infect Dis 25: 763781, 1997.

3. Brydon HL, Akinwunmi J, Selway R, Ul-Haq I. Brain abscesses associated with pulmonary arteriovenous malformations. Br J Neurosurg 13: 265-269, 1999.

4. Kawamata T, Takeshita M, Ishizuka N, Hori T. Patent foramen ovale as a possible risk factor for cryptogenic brain abscess. Report of two cases. Neurosurgery 49: 204-207, 2001.

5. Schuchlenz HW, Saurer G, Weihs W, Rehak P. Persisting eustachian valve in adults: relation to patent foramen ovale and cerebrovascular events. J Am Soc Echocardiogr 17: 231-233, 2004.

6. Rigatelli G, Dell'avvocata F, Braggion G, Giordan M, Chinaglia M, Cardaioli P. Persistent venous valves correlate with increased shunt and multiple preceding cryptogenic embolic events in patients with patent foramen ovale: an intracardiac echocardiographic study. Catheter Cardiovasc Interv 72: 973-976, 2008.
7. Kato Y, Dembo T, Takeda H, Fukuoka T, Tanahashi N. Prominent persisting Eustachian valve initiates spontaneous right-to-left shunt and paradoxical embolism in a patient with patent foramen ovale. Neurol Sci 32: 925-926, 2011.

8. Friedlander RM, Gonzalez RG, Afridi NA, Pfannl R. Case records of the Massachusetts General Hospital. Weekly clinicopathological exercises. Case 16-2003. A 58-year-old woman with left-sided weakness and a right frontal brain mass. $\mathrm{N}$ Engl $\mathrm{J}$ Med 348: 2125-2132, 2003.

9. Doepp F, Schreiber SJ, Wandinger KP, Trendelenburg G, Valdueza JM. Multiple brain abscesses following surgical treatment of a perianal abscess. Clin Neurol Neurosurg 108: 187-190, 2006.

10. Khouzam RN, El-Dokla AM, Menkes DL. Undiagnosed patent foramen ovale presenting as a cryptogenic brain abscess: case report and review of the literature. Heart Lung 35: 108-111, 2006.

11. LaBarbera M, Berkowitz MJ, Shah A, Slater J. Percutaneous PFO closure for the prevention of recurrent brain abscess. Catheter Cardiovasc Interv 68: 957-960, 2006.

12. Stathopoulos GT, Mandila CG, Koukoulitsios GV, Katsarelis NG, Pedonomos M, Karabinis A. Adult brain abscess associated with patent foramen ovale: a case report. J Med Case Reports 1: 68, 2007.

13. Chen FC, Tseng YZ, Wu SP, Shen TY, Yang HP, Chen CC. Vegetation on patent foramen ovale presenting as a cryptogenic brain abscess. Int J Cardiol 124: e49-e50, 2008.

14. Keith-Rokosh J, Hussain Z, Haig A, et al. Cryptogenic methicillin-resistant Staphylococcus aureus brain abscess. Can J Neurol Sci 35: 115-118, 2008.

15. Ariyaratnam S, Gajendragadkar PR, Dickinson RJ, et al. Liver and brain abscess caused by Aggregatibacter paraphrophilus in association with a large patent foramen ovale: a case report. J Med Case Reports 4: 69, 2010.

16. Lockhart PB, Brennan MT, Sasser HC, Fox PC, Paster BJ, Bahrani-Mougeot FK. Bacteremia associated with tooth brushing and dental extraction. Circulation 117: 3118-3125, 2008.

17. Berg L, Klebanoff LM. Focal infections. In: Merritt's Textbook of Neurology. 9th ed. Rowland LP, Ed. Williams and Wilkins, Baltimore, 1995: 136-142.

18. Yater WM. Variations and anomalies of the venous valves of the right atrium of the human heart. Arch Pathol 7: 418-441, 1929.

19. Bashour T, Kabbani S, Saalouke M, Cheng TO. Persistent Eustachian valve causing severe cyanosis in atrial septal defect with normal right heart pressures. Angiology 34: 79-83, 1983.

20. Douchette J, Knoblich R. Persistent right valve of the sinus venosus. Arch Pathol 75: 105-112, 1963.

(C) 2012 The Japanese Society of Internal Medicine http://www.naika.or.jp/imindex.html 